\section{Thyroid incidentalomas and the overdiagnosis conundrum}

\author{
“The problem of rising thyroid cancer rates represents a conundrum of \\ uncertainty with diagnosis and weighing the risks and benefits of \\ treating someone for something that may or may not pose a risk to that \\ person in the future."
}

\author{
Raymon H Grogan*,1, Briseis Aschebrook-Kilfoy², Michael G White', \\ Edwin L Kaplan' \& Peter Angelos'
}

First draft submitted: 29 March 2016; Accepted for publication: 29 March 2016; Published online: 14 July 2016

An incidentaloma is any mass or lesion discovered on a medical imaging study done for a reason unrelated to the organ where the incidentaloma is found. By definition, incidentalomas are asymptomatic and unsuspected by the physician ordering the initial study. As imaging technology improves and its use expands, it is logical to assume that incidentaloma detection rates will continue to increase. This is particularly concerning for an organ like the thyroid, because thyroid nodules are so common. It is estimated that up to $30 \%$ of the population has a thyroid nodule, which is roughly 90 million people in the USA [1]. When combined with the 80-100 million CT scans done annually in the USA one begins to get a sense of the potential problem. Simple probability suggests that roughly one out of every ten CT scans should yield a thyroid incidentaloma, which equates to 10 million thyroid incidentalomas per year in the USA. But, this is of course overly simplified math. This simple formula does not take into account the fact that many of those CTs do not image the neck. Nor does it consider the multiple other types of imaging modalities that do image the neck including MRI, ultrasound and PET. In addition, there is no way to know how many of those imaging studies were done specifically looking for thyroid pathology. And how do we know if the nodule was even reported by the radiologist, or worked up after it was reported? This thought experiment leads one to the realization that the problem of incidentally detected thyroid nodules is potentially enormous. However, determining how many thyroid nodules are found incidentally is difficult using probability theory alone because there are too many unknown variables. There are several questions that remain unanswered, but perhaps the most salient question currently is: How does the issue of incidentalomas relate to the problem of overdiagnosis of thyroid cancer?

The connection between thyroid incidentalomas and the overdiagnosis of thyroid cancer is perhaps the most important consideration facing the endocrine world today. Thyroid cancer incidence has increased worldwide at an exponential rate over the last three decades [2,3]. A clear cause for the rise in thyroid cancer incidence has yet to be delineated. However, research and scientific debate have given rise to two competing but not necessarily contradictory theories that attempt

'Endocrine Surgery Research Group, Department of Surgery, University of Chicago, 5841 S Maryland Ave MC 4052, Chicago, IL 60637, USA

2Department of Public Health Sciences, University of Chicago, 5841 S Maryland Ave MC N109, Chicago, IL 60637, USA *Author for correspondence: Tel.: +1 773702 7125; Fax: +1 773834 5295; rgrogan@surgery.bsd.uchicago.edu

\section{KEYWORDS}

- detection • incidentaloma

- overdiagnosis $\bullet$ thyroid cancer

- thyroid nodule 
to explain the increase in thyroid cancer incidence [4]. The first hypothesizes the increase in thyroid cancer is caused by a true increase in the incidence of thyroid cancers. This theory suggests that there has been a biologic change or fundamental shift in the etiology of thyroid cancer, and leads to inquiry into possible environmental exposures on a world-wide scale $[5,6]$. If this were true, then it would be incumbent upon the medical/scientific community to invest time and resources into identifying these environmental exposures in order to affect change on the rate of thyroid cancer diagnosis. The second theory suggests there is not a true increase in the incidence of thyroid cancer itself, but instead there has only been an increase in the incidence of the diagnosis of thyroid cancers. This theory rests on the premise that these cancers have always been in the population pool, but were simply overlooked in the past, but new and increased imaging has begun to identify these previously occult tumors. This leads to inquiry into the mechanisms of diagnosis of thyroid cancer, rather than the biologic etiology of thyroid cancer, and relies heavily on the idea that new imaging technologies are the main source of the rising rates. The end conclusion of this hypothesis is that the rising thyroid cancer rates are due to overdiagnosis [7]. In this scenario, resources should not be used to understand how to stop thyroid cancer from occurring, but instead should be used to find ways to stop diagnosing clinically silent thyroid cancers. It is also suggested that given the number of thyroid nodules in the population, thyroid incidentaloma detection is a significant contributor to the overdiagnosis problem. Casual perusal of the literature can be confusing as both hypotheses are represented, and adding to this confusion is a recent interest and coverage of the phenomenon in the lay press. Interestingly, this debate shares a striking resemblance to scientific debate in the 1940 s over increases in lung cancer incidence [8]. Regarding the modern day rise in thyroid cancer incidence, an in-depth look at the current body of scientific evidence reveals a certain level of equipoise, such that it is likely that there is validity to both sides of this debate.

Given the large number of thyroid nodules in the population, the connection between incidentalomas and rising thyroid cancer rates is plausible. However, if in routine clinical practice radiologists do not report every thyroid nodule that appears on every imaging study, then the true rate of thyroid incidentalomas does not approach the rate of thyroid nodules in the population. Additionally even if it is reported, it is still possible that in the greater context of the patient's medical care, nothing may be done about it. The majority of thyroid incidentaloma studies to date have relied on dedicated retrospective reviews of imaging studies in which radiologists go back and specifically look for incidentalomas and then report the incidence. This is not a reflection of the clinically important incidentaloma rate, it is more a reflection of thyroid nodule prevalence in the population. Additionally, few studies have looked at what happens to these incidentalomas after they are reported, that is, are they followed-up with a biopsy, and if so what is the final oncologic outcome?

Our research group recently undertook a study to better quantify the true contribution of thyroid incidentalomas to the rising rates of thyroid cancer [9]. We screened nearly 100,000 radiology reports of imaging studies done of the head, neck and chest with a computer algorithm to determine how many of those reports mention a thyroid nodule. We then went back and examined each of the reports identified to determine if the imaging study was performed for a reason other than specifically looking for thyroid pathology, thus identifying the thyroid nodule as an incidentaloma. We then went forward in the medical record to determine what happened to these incidentalomas in clinical practice. We found a very different picture from what has previously been described in the literature. Only $0.4 \%$ of imaging studies of the head and neck resulted in a radiologist reporting an incidental thyroid nodule and only $0.03 \%$ of studies resulted in a confirmed diagnosis of thyroid cancer [9]. Interestingly a second study done at an institution in a completely different geographic region of the USA also reviewed roughly 100,000 imaging studies and found a thyroid incidentaloma reporting prevalence of $0.1 \%$ [10]. This is strikingly similar to our finding and is also in stark contrast to previous studies that estimate the number of thyroid incidentalomas on imaging studies to be between 6 and $33 \%[1,11,12]$. An incidentaloma has no clinical significance if nothing is done about it, which appears to be the case for the vast majority of thyroid incidentalomas. The incongruity between the biologic thyroid incidentaloma prevalence and radiologists' actual rate of incidentaloma reporting suggests that the magnitude of incidentalomas' contribution to the increasing thyroid cancer incidence is much 
less than previously hypothesized. While this does not disprove the concept of overdiagnosis in thyroid cancer, it should at least call for a closer look at the purported cause of the overdiagnosis theory. Perhaps even more importantly, our results call for action regarding the potential for an as yet unknown cause of the increasing rates of thyroid cancer.

This finding is important because there has been a suggestion that overdiagnosis is caused in part by doctors 'looking too hard for thyroid cancer' [7]. The underlying implication of this suggestion is that if doctors just stop looking for thyroid cancer, the epidemic will disappear [13]. The incidentaloma conundrum shows that this just is not true. The truth is that incidentalomas are not as large a source of thyroid cancer diagnosis as some might believe, and doctors are not looking for thyroid cancer nearly as hard as some have suggested. So the idea that if doctors just stop looking for thyroid cancer that it will just go away is false, and for some patients is simply dangerous. As already suggested by a prominent thyroidologist, thyroid cancer is not some fiction created by doctors to line their pockets or prevent themselves from being sued [14]. The problem is not really overdiagnosis, the problem is one of inaccurate diagnosis. This should not be confused with incorrect diagnosis. The simple fact is that doctors have no way of reliably knowing which thyroid cancer will cause a problem and which will not. Furthermore, physicians practicing clinical medicine are not population scientists; they treat individual patients, one person at a time. When someone comes to a physician for advice that patient expects, rightly so, to be given personalized advice about their particular situation, not advice on how to potentially sacrifice their personal health for the well-being

\section{References}

1 Reiners C, Wegscheider K, Schicha H et al. Prevalence of thyroid disorders in the working population of Germany: ultrasonography screening in 96,278 unselected employees. Thyroid 14(11), 926-932 (2004).

2 Aschebrook-Kilfoy B, Schechter RB, Shih Y-CT et al. The clinical and economic burden of a sustained increase in thyroid cancer incidence. Cancer Epidemiol. Biomarkers Prev. 22(7), 1252-1259 (2013).

3 Kilfoy BA, Zheng T, Holford TR et al. International patterns and trends in thyroid cancer incidence, 1973-2002. Cancer Causes Control. 20(5), 525-531 (2009).

of the rest of the population. The answer is not to stop looking for thyroid cancer. The answer lies in figuring out which thyroid nodules and cancers are going to cause an oncologic problem and which ones are not.

Unfortunately, the technology to definitively determine the 'good' from the 'bad' thyroid cancers does not appear to be on the horizon. Thus doctors must do the best that they can to provide adequate care while avoiding harm if possible. Promising data from Japan suggest that observation of very small thyroid cancers may be reasonable. Other centers have begun trials to replicate these results. Additionally, recent American Thyroid Association guidelines have also begun to acknowledge that very small thyroid cancers may be treated with less than total thyroidectomy, and nodules under $1 \mathrm{~cm}$ in size may not need biopsy. The problem of rising thyroid cancer rates represents a conundrum of uncertainty with diagnosis and weighing the risks and benefits of treating someone for something that may or may not pose a risk to that person in the future. On its surface the problem may seem simple, but when faced with making decisions that could have life or death consequences for an individual, the reality becomes much more complicated.

\section{Financial \& competing interests disclosure}

The authors have no relevant affliations or financial involvement with any organization or entity with a financial interest in or financial conflict with the subject matter or materials discussed in the manuscript. This includes employment, consultancies, honoraria, stock ownership or options, expert testimony, grants or patents received or pending, or royalties.

No writing assistance was utilized in the production of this manuscript.

4 Davies L, Morris LGT, Haymart M et al. American Association of Clinical Endocrinologists and American College of Endocrinology Disease State clinical review: the increasing incidence of thyroid cancer. Endocr. Pract. 21(6), 686-696 (2015).

5 Zhang Y, Chen Y, Huang H et al. Diagnostic radiography exposure increases the risk for thyroid microcarcinoma: a population-based case-control study. Eur. J. Cancer Prev. 24(5), 439-446 (2015).

6 Ba Y, Huang H, Lerro CC et al. Occupation and thyroid cancer: a population-based, case-control study in Connecticut. J. Occup. Environ. Med. 58(3), 299-305 (2016).
7 Davies L, Welch HG. Current thyroid cancer trends in the United States. JAMA 140(4), 317-322 (2014).

8 Doll R, Hill AB. Smoking and carcinoma of the lung: preliminary report. $B M J 2$ (4682), 739-748 (1950).

9 Uppal A, White MG, Nagar S et al. Benign and malignant thyroid incidentalomas are rare in routine clinical practice: a review of 97,908 imaging studies. Cancer Epidemiol. Biomarkers Prev. 24(9), 1327-1331 (2015).

10 Davies L, Pichiotino E, Black WC, Tosteson AN. Developing a registry for thyroid incidentalomas: lessons learned and the path forward. Thyroid 26(5), 650-656 (2016). 
EDITORIAL Grogan, Aschebrook-Kilfoy, White, Kaplan \& Angelos

11 Youserm DM, Huang T, Loevner LA, Langlotz CP. Clinical and economic impact of incidental thyroid lesions found with CT and MR. AJNR Am. J. Neuroradiol. 18(8), 1423-1428 (1997).

12 Kang HW, No JH, Chung JH et al. Prevalence, clinical and ultrasonographic characteristics of thyroid incidentalomas. Thyroid 14(1), 29-33 (2004).

13 Hoang JK, Nguyen XV, Davies L. Overdiagnosis of thyroid cancer. Acad. Radiol. 22(8), 1024-1029 (2015).
14 Overdiagnosis in thyroid cancer: the case for observation. An Interview with R. Michael Tuttle, MD.

www.medscape.com/viewarticle/849893 